\title{
Easy \\ Subliminal Communication is Rossibte Using the DSA
}

\author{
Gustavus J. Simmons \\ Sandia Park. NM 87047. USA
}

\begin{abstract}
In 1985, Simmons showed how to embed a subliminal channel in digital signatures created using the El Gamal signature scheme. This channel, though, had several shortcomings. In order for the subliminal receiver to be able to recover the subliminal message, it was necessary for him to know the transmitter's secret key. This meant that the subliminal receiver had the capability to utter undetectable forgerjes of the transmitter's signature. Also, only a fraction of the number of messages that the channel could accommodate in principal could actually be communicated subliminally $(p(p-1)$ messages instead of $p-1)$ and some of those that sould be transmitted were computationally infeasible for the subliminal receiver to recover.
\end{abstract}

In August 1991, the U.S. National Institute of Standards and Technology proposed as a standard a digital signature algorithm (DSA) derived from the El Gamal scheme. The DSA accommodates a number of subliminal channels that avoid all of the shortcomings encountered in the El Gamal scheme. In fairness, it should be mentioned that not all are avoided at the same time. The channel in the DSA analogous to the one Simmons demonstrated in the El Gamal scheme can use all of the bits contained in the signature that are not used to provide for the security of the signature against forgery, alteration or transplantation, and is hence said to be broadband. All messages can be easily encoded for communication through this channel and are easily decoded by the subliminal receiver. However, this broadband channel still requires that the subliminal receiver know the transmitter's secret key. There are two narrowband subliminal channels in the DSA, though, that do not give the subliminal receiver any better chance of forging the transmitter's signature than an outsider has. The price one pays to secure this integrity for the transmitter's signature is a greatly reduced bandwidth for the subliminal channel and a large, but feasible-dependent on the bandwidth actually used-amount of computation needed to use the channel. In one realization of a narrowband subliminal channel, the computational burden is almost entirely on the transmitter while in the other it is almost entirely on the subliminal receiver.

In this paper we discuss only the broadband channel. The narrowband channels have been described by Simmons in a paper presented at the 3rd Symposium on State and Progress of Research in Cryptography, Rome, Italy, February 15-16, 1993. Space does not permit them to be described here. The reader who wishes to see just how easy it is to communicate subliminally using the DSA is referred to that paper as well. The inescapable conclusion, though, is that the DSA provides the most hospitable setting for subliminal communications discovered to date.

\section{Prologue}

One of the Inevitable consequences of the development of digital signature standards such as the digital signature algorithm (DSA) proposed by the U.S. National Institute of Standards of Technology [2,3] will be their application to the authentication of documents, licenses, personal ID's, etc. Because a widely -even universally-accepted standard can be used, it will be possible for anyone, using only public information and the information contained in the signed instrument, to verify that that information could only have been signed by the specified issuing authority. The verifier can therefore be confident that the 1ssuer is vouching for the integrity of the information he signed; 1.e.. that the Issuer has Independently established the Identity of the bearer of the docu. ment and that he has verified the license, authority, credit or other information vouchsafed for in the dacwent. As a result, any customs checkpoint in the world will be able to quickly verify the integrity of the information contained 
in a passport-including the photograph of the person to whom it was issued-and hence to determine whether it is being presented by its legitimate owner. Similarly, a teller at a bank, a clerk in a store, a guard at a restricted access facility, etc., will be able to validate identifying and authorizing information in personal ID's, licences, etc. There is no doubt that this is both possible and probable as a result of the development of widely accepted and easy to implement digital signature standards.

What Isn't obvious, though, is that some digital signature schemes, and the DSA in particular, make it easy to conceal information in the signature which can be recovered by insiders, $1 . e$, by persons possessing information about the scheme that was not made public, but which can't be recovered or whose presence can't even be detected by persons possessing only the public information. Information communicated in this way-indetectably concealed in a public communication-1s said to be subliminal, and the mechanism for its communication is called a subliminal channel. The net result is that the digital signature on a passport which makes it possible for anyone who wishes to verify the authenticity of the document can also tell customs agents of the issuing nation that the bearer is a known terrorist, drug dealer, smuggler or felon. The digital signature on the driver's 1icense that verifies the bearer's identity to the merchant or teller can tell law enforcement officers the bearer's driving while intoxicated (DWI) record, or his accident or traffic violations history, etc. Even commercial entities wight have an interest in exploiting such a concealed channel. A financial institution issuing credit cards that are also used as personal ID's might well wish to conceal in the digital signature information about the customer's credit rating, payment history, etc., that could only be read by their agents.

The fact that subliminal messages can be concealed in digital signatures is not a new observation. What is new is that the shortcomings whlch limited the practical feasibllity of earlier schemes can all be avolded in subliminal channels in the DSA.

\section{Introduction}

In 1985 Simmons [4] showed that in any digltal signature scheme in which a bits are used to communicate a signature that provides only $\beta$ bits of security against forgery, alteration or transplantation of a legitimate signature, where $\beta<\alpha$, the remaining $\alpha-\beta$ blts are potentially available for subliminal comminication. However, the subliminal channels that have been found in most such digital signature schemes suffer from a number of deficiencies. If the subliminal channel uses $a l l$, or nearly $a l l$, of the $\alpha-\beta$ bits, it is sald to be broadband, while if it can communicate only very small fraction of the $a-\beta$ bits, it is said to be narrowband. All broadband channels devised thus far suffer from a common, and serious, shortcoming: the subliminal recalver must 
know the transmitter's secret signing key in order to be able to recover the subliminal message(s). This means that the subliminal recelver has the capability to utter undetectable forgeries of the transmitter's signature. While there are situations in which a transmitter is willing to unconditionally trust the subliminal receiver, there are many more in which he isn't. Also in most cases, not all of the $\alpha-\beta$ bits available can actually be used for subliminal communications. Put another way, in a natural sense not all messages can be encoded for communication through the subliminal channel-and much more significantly not all of those that can be encoded can be recovered by the subliminal recelver. This is due to the fact that the decoding algorithm in most cases requires an extremely variable amount of computation by the subliminal receiver -ranging from the computationally trivial to the infeasible-depending on which message(s) are being decoded.

Subliminal channels that do not require the transmitter to entrust his secret key to the subliminal recelver are generally narrowband. The reason for this is that the security for the transmitter's signature against forgery by the subliminal recelver is bought at the expense of requiring at least one of the two to do computations that grow exponentially with the number of bits being communicated subliminally. Simmons has described two of the narrowband channels in the DSA at the 3rd Symposium on State and Progress of Research in Cryptography, Rome. Italy, February 15-16, 1993 [6]. In this paper, however, we will concentrate on the broadband channel that can be set up between a transmitter and subliminal receivers whom he trusts unconditionally since this is the most efficient (in an information theoretic sense) of the several channels peraitted by the DSA.

\section{A sublininal channel in the 81 Ganal DB8}

The subliminal channel that Simmons discovered in the El Gamal digital signature schemel - from which the DSA is derived-clearly 1llustrates all of the deficlencies mentioned above [5], so we start by discussing this channel in greater detall than has been done before in order to set the stage for discussion of subliminal communications using the DSA. The likelthood of the DSA being adopted as a digital signature standard (DSS) by the U.S. makes such a discussion particularly timely and important; especially given the truly remarkable coincidence that the subliminal channels in the DSA avold every one of the deficiencies mentioned above. In fairness, it should be said that no single channel (known to the author) avoids all of them simultaneously, however. several channels avoid all but a single one of them.

simmons observed that if the transmitter ${ }^{2}, T_{x}$, shares his secret signing key, $x$, with another person-the subliminal receiver, $s_{x}$-this person will under

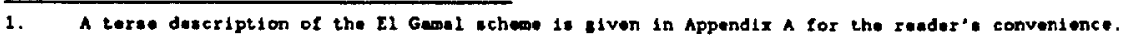

2. In order to otreaine the discusaion, the signer of user wo orisinetes end/or tranamits the digitally

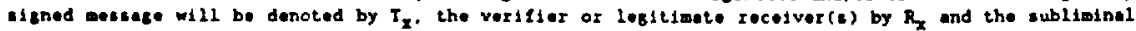
rece1ver(s) by $s_{x}$. 
some clrcumstances be able to recover the secret session key, $k$, used by the $T_{x}$ to generate the digical signature. When this is possible, the $I_{x}$ can encode the subliminal message in the choice of $k$-which as we will show can only be recovered by someone in possession of the $T_{x}$ 's secret key, $x$. This, of course, requires that the $T_{x}$ trust the $S_{x}$ unconditionally since the $S_{x}$ will have the capability to utter undetectable forgeries of the $T_{x}$ 's signature. A common reaction at this point is to say that no one in their right mind would accept such a condition, 1.e., no one would unconditionally trust someone else with what amounts to a "power of attorney" for their signature. It should be pointed out, however, that all participants in single-key cryptosystems have always had to accept precisely this condition since any party can do anything the others can. In other words, it has only been since the discovery of public key (twokey) cryptography that participants have even had the option of having sowe degree of security for their communications without having to unconditionally trust the integrity of the other party(les) to the communication.

Briefly, the subliminal channel in the $E I$ Gamal digital signature scheme works as follows. There is an overt message m which the $I_{x}$ signs. Since may be quite lengthy, it is first hashed using a publicly known hashing function $H(\cdot)$ whose range is contained in GF(P); the fleld over which the El Gamal signatures are computed. The signature is actually for $h-H(m)$, but since H(*) is chosen to be atrong one-way hashing function it is infeasible (1mpossible?) for anyone given a message $\mathbf{m}$ to find another message $\mathbf{w}^{\prime}$ such that $H(m)-H\left(m^{\prime}\right)$, or given an $h$ to find an wuch that $H(m)-h$. Consequently, signing $h$ is practically the same as signing $m$. The signed message is the triple $(m ; r, s)$ where

and

$$
r=\alpha^{k} \bmod p
$$

$$
s=k^{-1}(h-x r) \bmod (p-1)
$$

$X$ is the $T_{X}$ 's secret signing key and $k$ is a session key-supposedly randomly chosen from the Interval $0<k<p$ such that $(k, p-1)-1$ - to be used only in the generation of the signature for $m$.

The verification procedure for the signature is of no concern here. Suffice it to say that it is insensitive to the particular choice of $k$, so long as the conditions $0<k<p$ and $(k, p-1)-1$ are satisfled.

Now consider what the $s_{x}$ can do by virtue of knowing $x$, that the public receivers, $R_{X}$, cannot do. Everyone knows $p$, a (a primitive element in $G F(p)$ ), the $T_{x}$ 's public key $y=a^{x} \bmod p$, the hashing function $H(\cdot)$ and the signed message $(w ; r, s)$. Also, they can easily compute $h-H(m)$. The $S_{x}$, though, can compute 


$$
w-h=x r \bmod (p-1)
$$

It is easy to see, and to show, that for the $R_{x}$, is unconditionally securely encrypted by the $T_{x}$ 's secret key, $x$. In other words knowing both $h$ and $r$, the $R_{X}$ 's uncertainty about the value of $w$ is precisely what it would be if he knew neither $h$ nor $r$. The $s_{x}$ knows from (2) that

$$
s=k^{-1} w \bmod (p-1) \text {. }
$$

$\left(k^{-1}, p-1\right)-1$ since $(k, p-1)=1$, so that

$$
E=(s, p-1)=(w, p-1) \text {. }
$$

If the $S_{X}$ is lucky, $f=1$ and (4) can be easily solved to recover $k$ directly,

$$
k=s^{-1} \mathbf{s} \bmod (p-1)
$$

With probability $1-\varphi(p-1) /(p-1)>1 / 2$, though, $f>1$ so that $s$ does not have a multiplicative inverse with respect to $p-1$. The communications channel we have just described is a rather strange one. There are p-1 possible values that $k$ could take, 1 .e., messages that conceivably might be communicated sublimInally, but since $(h, p-1)-1$, only $\varphi(p-1)$ of these can actually be encoded. Given that one of the permissable $k$ is encoded, we have fust seen that due to the randow mapping effect of the $T_{x}$ 's secret key which was chosen in advance and is fixed thereafter-that the probability that $k$ can be recovered using the simple decoding scheme in (5) is $f(p-1) /(p-1)<1 / 2$. This is the subliminal channel in the El Gamal digital signature scheme as Simmons described it. As we shall see there is a great deal more to be said about this simple scheme.

If $(s, p-1)=f>1$ so that $s$ does not have a multiplicative Inverse, we can still solve for $k$ in almost all cases using the forward search cryptoanalytic technique devised by Holdridge and simmons [7] as a means to attack a public key secrecy channel in those cases in which the source equivocation is swall enough to make an exhaustive search feasible. In such situations, even if the encryption operation is impossible to invert, since the encryption key is public it is possible for the cxyptanalyst to preencrypt plaintexts to generate a table of plaintext/ciphertext pairs, and then use table lookup to find the plaintext corresponding to a ciphertext-even though direct decryption is impossible. In the application being discussed here, inverting $r-\alpha^{k}$ to recover $k$ directly is the hard problem of taking discrece logarithms (with respect to $\alpha$ ). We can, however, In almost all instances, establish that $k$ is an element in a set $x$ whose cardinality is small enough that it is feasible to calculate the wodular exponentiation, $\alpha^{k_{1}}$, for $a l l k_{1}<x$. It is then an easy matter to identify $k$ 1tself.

To carry out a forward search for the session key, $k$, when $(s, p \cdot 1)>1$, we reduce (2) to form 
where

$$
u=\left(\frac{s}{f}\right)^{-1}\left(\frac{h-x r}{f}\right) \bmod \left(\frac{p-1}{f}\right)
$$

$$
\left(\frac{s}{f}\right)\left(\frac{s}{f}\right)^{-1}=1 \bmod \left(\frac{p-1}{f}\right) \text {. }
$$

We now know that $k$ is a unique member of the set

$$
\pi-\left\{u+i\left(\frac{p-1}{f}\right)\right\} \quad 0 \leq i<f .
$$

$k$ Itself can be found by calculating $r_{1}=a^{z_{1}} \bmod p_{,} k_{1}<2$, to identify the element of $X$ that reproduces $r$.

If $f$ is small, the forward search is computationally easy. For large values of $f$, however, forward search may be infeasible. A result from elementary number theory states that if $d$ is a divisor of $N$, the number of positive integers, $J<N$, for which $(J, N)-d$ is $\varphi(N / d)$. In the present case, $N-p-1$, so that for $\varphi(p-1)$ values of $r, k$ can be recovered by the $s_{x}$ without having to resort to forward search. If $d / N, d>1$, then on average $(d+1) / 2-1 / d$ encryptions must be made before $k$ is identifled. We will later examine this result more closely. but for the moment, consider what it says about the difficulty of recovering the session key, $k$, from a knowledge of the secret key, $x$, if the prime $p$ is of the form $p-2 p^{\prime}+1$. $\varphi(p)-2 p^{\prime}$ in this case so that in $p^{\prime}-1$ cases $k$ could be recovered using (5). In $p^{\prime}-1$ cases $f-2$, so that precisely one modular exponentiation would need to be done to identify $k$, and in the one case in which $E$ - $p^{\prime}$, It would be computationally infeasible to use forward search to recover $k$. The statement that $k$ can be recovered in almost all cases from a knowledge of $x$ using forward search techniques is thus justified for $p$ of the form $p-2 p^{\prime}+1$. The generalization to other $p$ is obvious-but tedious.

To be precise, what we have called forward search-although closely related to the technique devised by Simmons and Holdridge-isn't truly forward search. Furthermore, the computational effort required to decode subliminal messages can be considerably less than indicated in the naive approach described. We defer a discussion of these points for the moment to exhiblt a modest sized example illustrating all aspects of the subliminal channel.

Let $p=4294969663$ so that $p-1-2 \cdot 3 \cdot 715828277$. 17 is a primitive element in $G F(p)$, so we set $a-17$. p was chosen to be a ten-digit prime to make it possible to send four-letter words as subliminal messages. There is no secure hashing function over such a small range so we use a simple check sum as a message digest algorlthm to compress arbitrarily long texts into 32 -bit digests. The message digest $H(m)$ will be the exclusive or of successive blocks of 32 -bits of the binary encoded text. This is especially conventent if the text is encoded Into 8-bit ASCII, since then the blocks each contain four characters, padded with blocks of 8 zeroes, 9 , if need be to make the text be a multiple of 32 bits. The hash of the text "NOT SEEING IS BELIEVING" then becomes. 
or

$($ NOTsp $) \oplus($ SEEI $) \oplus($ NGspI $) \oplus($ SspBE) $\oplus($ LIEV) $\oplus$ (INGg)

$$
h=H(m)-(101111010101111000100110011)_{2}-99283251 \text {. }
$$

Since $\varphi(p+1) /(p-1)=1 / 3$ in this case, assuming that the ASCII encoding of fourletter words is a random mapping with respect to relative primality to $p-1,0$ ily one out of three four-letter words has an ASCII code that is sultable for sub-

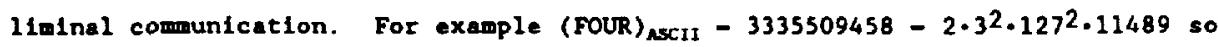
that ("FOUR," p-1) - 6 which says that the message "FouR" can't be communicated sublintnally, while (FORE) asciI - 3335508677 (a prime) so that "FORE" could be.

Assume also that the $T_{X}$ 's secret key, $X, 1 s 2045235856$ and is known to the $s_{x}$. The $T_{x} s$ public key, $y-a^{x}$ mod $p$, isn't needed for the subliminal commication. One signature for the message NOT SEEING IS BELIEVING would be

(NOT SEEING IS BELIEVING; 870994817, 959793649)

The $S_{x}$ on receiving this signed message would first calculate (s,p-1) - 1 which tells him that he can solve for $k$ directly using ( 5 ). He then calculates $s^{-1}-1794768229$ and

Final1y

$$
\begin{aligned}
w=h-x r & =326880830 s \bmod (p-1) \\
k=w s^{-1} & =3335308741 \bmod (p-1) \\
& =(11000110110011001100010111000101)_{2}
\end{aligned}
$$

which is the ASCII encoding of the subliminal message "FLEE."

As we've mentioned earlier, the verification of the signature-by either the $R_{X}$ or the $S_{X}$-is unaffected by the presence of the subliminal communication. If the messages, both overt and covert, remain the same, and the same prime and primitive element are used-which means, of course, that $r=a^{k}$ mod $p$ also remains the same, but the $T_{x}$ 's secret key, $x$, is smaller by only 1 . 1.e., $x-2045235855$ instead of 2045235856 the result is quite different. $s-2985295794-2 \cdot 3 \cdot 13 \cdot 38273023$ so that $f=(s, p-1)-6$. The $S_{x}$ calculates $w=4139803122$ and knows that he must use (6) to solve for $u$.

$$
\begin{aligned}
& u=(497549299)-1 \cdot 689967187 \bmod \left(\frac{p-1}{6}\right) \\
& u=471995633 .
\end{aligned}
$$

He therefore knows that the subliminal message is the unique element, $k_{1}$, in the set

$$
X=\{471995633+1 \cdot 715828277 \bmod (p-1)) \quad 0 \leq 1 \leq 5
$$

for which $a^{k_{1}}-r \bmod p$.

One way to find $k$ would be to calculate the modular exponentiations $a^{k}$ mod $p$ until $r$ is recovered and $k$ ldentified. Thls would require a large amount of computation, almost all of which would be wasted. A better approach that actually uses the forward search cryptanalytic technique is to note that for some 1 . 


$$
r=a^{k}-a^{u+1(p-1) / f} \bmod p
$$

$u$ is determined by the subliminal communication, while (p-1)/f is simply a divisor of $p-1$. We rewrite (8) in the form

$$
r a^{-u},\left\{a^{1(p-1) / E} \bmod p ; 0 \leq 1 \leq 5\right\}-D_{6} .
$$

Let $d-a(p+1) / f \bmod p$, then we can simply form the powers of $d$ as opposed to computing full modular exponentiations.

$$
\operatorname{ra}^{-u} \in\left\{d^{1} \bmod p ; 0 \leq 1 \leq 5\right\}=D_{6}
$$

Df can now be precomputed as soon as $p$ is chosen. There are a number of time saving computational tricks to computing the various Df since sets will have elements in common whenever the divisors, $f$, have factors in common. The Important point is that $D_{f}$ is now a precomputed table $\left(1, d^{1} \bmod p\right)$ which is a true application of forward search. When the signed message $(m ; r, s)$ is received $f$ is calculated from $(s, p-1)$ using the Euclidean algorithewhich is only of order $0(\log (p))$ difficulty-and $u$ solved for using formula (5). ra-u is then calculated and looked up in the table to identify the correct value of $i$. Final1y

$$
k=u+i \frac{p-1}{f}
$$

is calculated to recover the subliminal message.

In the present example $D_{6}$ is given in Table $I$.

\begin{tabular}{ll} 
& Table I. \\
\hline$I$ & $d_{1}$ \\
0 & 1 \\
1 & 983925568 \\
2 & 983925567 \\
3 & 4294969662 \\
4 & 3311044095 \\
5 & 3311044096 \\
\hline
\end{tabular}

We next calculate

$$
a^{-u}=3040091830
$$

and

$$
a^{-u_{r}}=3311044095 \bmod p
$$

which is the fifth entry in the table; $1-4$. We can, therefore, finally decode the subliminal message

$$
k=471995633+4 \cdot 715828277-3335308741
$$

which we recognize from before as the ASCII encoding of the messege "FLEE." This is an efficient decoding algorithm for the subliminal channel. When a 
message is received, a single modular exponentiation is required to compute $\alpha^{-u}$ mod $p$ followed by a modular product and a table lookup. These steps are Independent of the value of $f$ which determines whether it is feasible to carry out the forward search calculations needed to precompute the table $D_{f}$ in the first place.

We have seen that if the user's secret key is $x-2045235856$ the subliminal message is directly recoverable without forward search, while if $x-x-1$ there are $s i x$ possible values for $k$-but that the equivocation can be resolved by a forward search to Identify the one that generates the known cipher, $r$. If $x-x-2$, however, $f-715828277$ so that the forward search would have to be carried out in a set of size 715828277. Although this is barely within the state of the art for such a small example, it illustrates the infeasibility of recovering the subliminal message when $f$ is a large divisor of $p-1$ for realistic sized primes, $p$, even though the message could be easily concealed and communicated in the subliminal channel. The reader may have guessed that our intial "random" cholce of $x-2045235856$ was not entirely random. In fact, it was chosen to illustrate the different levels of computational difficulty that can be Involved in carrying out a forward search for keys that are very nearly the same. Table II summarizes this dramatically. For the entries in this table, the message (NOT SEEING IS BELIEVING), the modulus (4294969663) and the prim1tive element, 17, are all fixed. The secret key, $x$, and parameters dependent on $x$ are variable. Since $r$ is a function of only fixed elements, it is also fixed at $r-870994817$ in all five cases.

Iable II.

\begin{tabular}{clc}
\hline$x$ & \multicolumn{1}{c}{$\boldsymbol{c}$} & $\boldsymbol{f}-(\mathrm{s}, \mathrm{p}-1)$ \\
2045235856 & 959793649 & 1 \\
2045235855 & 2985295794 & 6 \\
2045235854 & 715828277 & 715828277 \\
2045235853 & 2741330422 & 2 \\
2045235852 & 471862905 & 3 \\
\hline
\end{tabular}

The broadband subliminal channel in the El Gamal DSS has several deficiencies: some serious and some of only academic concern. The signature $(\cdot ; r, s)$ consists of $2 l$ bits, $l-\left\lceil\log _{2} p\right\rceil$. the equivocation of $l$ of which 1 s "used up" to provide a security of 2-l against forgery, alteration or transplantation of the signature. The remaining $l$ bits are potentially avallable for subliminal communlcations, however as we've seen, only $\log _{2}(\varphi(p-1))$ of these can be used. This says that one blt will be wasted in all cases, so natural question is how significant is this loss of channel capacity. It is easy to verify directly that $p-421$ is the smallest prime for which two bits are unavallable. This problem, however, Is of academic interest only, since a number theoretic calculetion shows that no prime with fewer than 71 decimal digits can lose as many as three bits and that the prime would have to have 7777 decimal digits in 
order for four bits to be unavailable! The conclusion is that for realistic sized primes only a bit or two out of the $l$ bits total will be unavailable for subliminal communications.

A somewhat more serious (practical) problem is that there is no natural encoding/decoding between the space of binary messages and the elements of $M_{p}$. As we've seen, we can only encode $k$ for which $(k, p \cdot 1)-1$ for communication in the subliminal channel, but we must have a practical way to associate these values of $k$ with the messages we wish to convey, which is easy for both the $T_{X}$ and the $S_{x}$ to use. For large primes, and especially for primes for which $p-1$ has several factors, this is so difficult to do that it limits the potential usefulness of the subliminal channel.

The next most important problem-in increasing order of significance-is the fact that some messages that can be easily encoded for communication in the subliminal channel are completely infeasible for the $s_{x}$ to recover but which message is lost will depend on the value of $H(m)$. Depending on the factorization of p-1, this may be a serious problem (because of the number of messages that can't be conveyed to the $s_{x}$ ) or a problem of only academic interest as is the case when $p=2 p^{\prime}+1$ where there is only a single message that can't be recovered by the $s_{x}$. It should be pointed out, however, that this "lost message" isn't fixed by the choice of $x$ so it can't be avolded by the coding scheme, rather for any cholce of the message $m$, and hence of $h$, and of $x$, there will be one message whose encoding w111 be infeasible for the $s_{x}$ to recover. For primes not of the special form $p-2 p^{\prime}+1$, the preceding comments apply to a larger set of nessages.

Finally, there is the most serlous deficiency of all; namely, that the $s_{x}$ must know the $T_{x}$ 's secret key, $x$, in order to recover the subliminal nessages.

\section{A sublininal channel in the D8A}

Since both components of the signature $(\cdot ; r, s)$ created using the DSA are elements in GF( $q), 2 l$ blts must be communicated with a message, $m$, to convey the signature; where $2-\left\lceil\log _{2} q\right\rceil$. If for any given message either $r$ or $s$ is fixed, the probability of a would-be cheater finding the unique value of $s$ or respectively, that would cause the triple $(m ; r, s)$ co be accepted as having been signed by the specified user is precisely the same as the probability of drawing a unique but unknown, element from $G F(q)$ in a random drawing, 1.e., 1/q. This statement holds, even though $r$ almost certainly cannot assume all of the values in $\mathrm{GF}(q)$. The concatenated modular reductions in forwing $r$

$$
r=\left(g^{k} \bmod p\right) \bmod q
$$

map the residues of $g^{k}$ mod $p$ onto residues mod $q$. Since $g$ was constructed to be an element of order $q$ in $G F(p)$, as $k$ ranges over the elements of $G F(q), g^{k} \bmod p$ ranges over a unique cycle of order $q$ in $G F(p)$. Note: the number of elements 
of order $d$, where $d / p-1$, In $G F(p)$ is $\varphi(d)$. Since $d$ is the prime $q$ in this case there are $q-1$ elements of order $q$ in $G F(p)$. This says that no matter which element of order $q$ is chosen as $g$, that as $k$ varies over $G F(q)$, the same set of $q$ elements (In permuted order of course depending on the choice of $g$ ) will be generated by $g^{k} \bmod p$. The residues mod $q$ in turn of these $q$ residues will be randow elements of GF( $q$ ).

For example, if we choose $p-547$ and $q-13$, the set of residues of $g^{k}$ mod $P, 8$ an element of order 13 in GF(p), is,

$$
(1,46,237,261,293,350,353,375,440,475,509,517,519) \text {. }
$$

The set of residues of this set $\bmod 13$, is,

$$
\left(1^{2}, 2^{2}, 3,7^{3}, 10,11^{2}, 12^{2}\right)
$$

where the superscripts indicate the multiplicitles with which the indicated $r$ occurs.

$$
46-293-475-7 \bmod 13,
$$

etc. Note that in this example, $r$ cannot be any one of the elements in the set $(0,4,5,6,8,9)$. The mapping of the $q$ residues of $g^{k}$ mod $p$ onto the residues nod $q$ is nodeled by the randon drawing of $q$ elements from a set of $q$ distinct objects with replacement. For $q$ large $\left(2^{16}\right.$ is large) the expected number of distinct elements in the drawing will be $q(1-1 / e)$. In other words, the probabillty that a randomly chosen $r, r, G F(q)$, can actually occur in a signature w11l be only $p=0.63$. On the other hand, it is easy to construct an $r$ that does occur. Choose any $k, G F(q)$ and compute $I=\left(g^{k} \bmod p\right) \bmod q$. Given any permissible $r$, all elements of $G F(q)$ are possible values for $s$ (depending on the values of $h, x$ and $k$. Conversely, given any value of $s$, every value of $r$ that can occur does occur (again depending on the values of $h, x$ and $k$ ). Since it is computationally infeasible to exhaustively calculate the values that $r$ can assume, and impossible to show that a particular value cannot occur except by an exhaustive search, the statement made earlier about the uncertainty of $r$ (given an $s$ ) or of (given an $r$ ) being $l$ bits is true. In other words, $l$ of the $2 l$ bits in the signature are expended to provide security for the signature.

The remaining $l$ bits are avallable for subliminal communications. We next show that $a l l$ of them can be used.

As was the case for the broadband subliminal channel in the El Gamal digital signature scheme, the $T_{x}$ shares his secret key, $x$, with the subliminal recelver(s) to set up the sublininal channel in the DSA as well. The object is for the $T_{x}$ to be able to communicate an arbitrary element of GF*(q) to the $S_{x}$ In such way that it will be impossible for anyone to efther recover the sublim-inal message $m^{\prime}$ without knowing $x$, or to detect that the subliminal channel is being used, even if he knows the subliminal wessage(s), m', being comnunicated. 


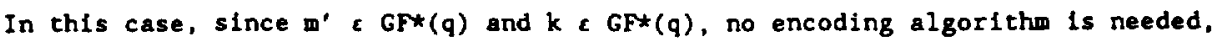
we can simply take $k$ to be the desired subliminal message, $k-w^{\prime}$. The Ix sends the overt signed message $(m ; r, s)$, where

and

$$
r=\left(g^{k} \bmod p\right) \bmod q \text {. }
$$

$$
s=k^{-1}(h \cdot x r) \bmod q \text {. }
$$

Since the modulus is a prime, any nonzero element of GF(q) has a multiplicative inverse. For the moment, assume that $w-h \cdot x r \sim 0$, so that $s, 0$, then

$$
k \cdot s^{-1} \mathbf{m o d} q
$$

as before. It is important to note that because the modulus is a priae in this case (it was p-1 in the El Gamal scheme, which vas necessarily composite) that $(s, q)-(w, q)-1$, so that forward search is never needed to decode a sublininal message. Since $k \in G F *(q) k \bullet 0$ and $k^{-1} \sim 0$, however, w can be 0 so that $s-0$ is posstble, in whlch case $k$ cannot be recovered. To see this, consider the same example used before: $p-547, q-13, g-475, h-6$ and $r(1,2,3,7$, 10, 11, 12). Let the $T_{x}^{\prime}$ s secret key $x-3$. $w-0$ if $r-2$ in this case, 1.e., If $k-3$ or 4 . What this says is that for this small example and for any message that hashes to produce a value of $h=6$, nefther $k-3$ nor $k-4$ can be communicated subliminally. The probability of $s$ being 0 , however, is $1 / q, 1 . e$. $\approx 2-160$, and even if it is 0 , the expected number of subliminal messages that cannot be communlcaced is only $e /(1-\mathrm{e}) \approx 1.58$. To appreciate these numbers: the age of the universe measured in microseconds from the $\mathrm{big}$ bang to the present is less than $2^{79}$. Thus, although there is a nonzero probability that an - c GF(q) cannot be communicated subliminally, the channel capacity is $l-c$, where $c<10.47$.

To sumarize, the broadband channel in the DSA is easy for the $T_{x}$ to encode for and for the $s_{x}$ to decode: forward search is never needed to resolve amblguities. All messages can be encoded-and more importantly all messages can be decoded. To be precise, the latter statement is only 1 -c true as discussed in the previous paragraph, but we wil not continue to qualify every statenent about the channel with this $c$ qualifier.

The channel just described does have a weakness, though. If anyone knows the subliminal message that the $T_{x}$ wishes to communicate, he can easily recover the $T_{X}$ 's secret signing key, $x$. First, anyone can compute $h$ using the public hashlng function $H(\cdot)$ and the overt communlcation $(m ; r, s)$. From (13), he knows that

$$
\mathbf{x}=\mathbf{r}^{-1}(\mathbf{k s - h )} \bmod \mathbf{q}
$$

If neither $r$ nor $k s \cdot h$ is 0 , so that with probability 1 - (1/q), 1.e., with probability 1 for all practical purposes, knowledge of the intended subliminal message equates to knowledge of the signers secret key. As a matter of fact, the uncertainty about the signer's secret key, $x$, is reduced by precisely the amount known about the session key, $k$, Since $x$ should pose $l$ bits of uncertainty to 
everyone except the $T_{x}$, this means that $k$ must be $l$ bits uncertain as well-even when the intended subliminal message is known, l.e., even when there is no uncertalnty at all about $\mathrm{w}^{\prime}$. Since $k$ is an element in the mulciplicative group $G F(q)$, it is easy to achieve this level of unconditional security. The $T_{x}$ and the $S_{x}$ secretiy share in advance ane-time key consisting of a random sequence, $v$, of symbols drawn from GF* $(q)$. They then use the next unused symbol, $v<v$, from this sequence to Vernam encrypt/decrype the subliminal message, $m^{\circ}$, using wuleiplication in $G F(q)$. To recover the text $\mathbf{m}^{\prime}, S_{x}$ first recovers $k$ as described above and then calculates $m^{\prime}-k v^{-1}$ in $G F(q)$. The concealment is perfect, Irrespective of how many known plaintext's the public recelver may have. since the Vernam encryption always introduces at least as much equivocation (per encryption) as is removed by the exposure of a clpher/ plaintext palr. The bottom line is that the broadband subliminal chennel in the DSA is perfectly concealed-even from an opponent who suspects its use and who knows exactly the messages being communicated through it.

Unconditional security for the subliminal messages and unconditional concealment of the fact that subliminal communications are occurring requires that the key sequence $V$ be a truly random sequence. For practical purposes the $I_{x}$ and $S_{x}$ would simply share the key to any cryptographically secure key stream generator-the synchronlzed output of which would be used instead of the onetime key sequence described here.

The broadband channel still suffers from the necessity that the $s_{x}$ know the $T_{x}$ 's secret signing key in order to be able to decode subliminal communication. Space doesn't permit a description of the narrowband subliminal channels allowed by the DSA that overcome this Iimitation. The interested reader is referred to a paper presented by simmons at the 3rd symposium on state and Progress of Research in Cryptography, Rome, Italy, February 15-16, 1993, for a detailed discussion of these channels.

\section{Conclusion}

The DSA provides the most hospltable setting for subliminal communications discovered to date.

\section{Note to the Appendices}

All digital signature schexes presuppose the existence of a secure hashing function $H(\cdot)$, which operates on messages to produce message digests. $H(\cdot)$ satisfles the condition that given a message $m$ it is computationally infeasible (Impossible?) to find another message $w^{\prime}$ such that $H(m)-H\left(m^{\prime}\right)$; $1 . e . H(\cdot)$ is collision free. Equivalenty, given an $h$, it is infeasible to find an $m$ such that $H(m)-h$. $H(\cdot)$ is public information, and for all messages $m, H(m)$ is easy to evaluate. 


\section{Appendix $\mathbf{A}$.}

The El Gamal digital signature scheme [1] involves four distinct steps.

Step 1 (performed by a trusted issuing authority).

- a large prime, $p$, is randomly selected, subject to the condition that p-1 has at least one large prime factor.

- a primitive element $a \in G F(p)$ is chosen.

Note: $p$ and a are made public and can be used by a comunity of users.

Step 2 (performed by each user who wishes to be able to sign messages).

- user chooses a randow $x \in G F^{\star}(p)$, which is his secret (signing) key.

- user publishes $y=a^{x} \bmod p$ as his public (verification) key.

Note: each user's public key, $y$, is associated with his identity in a pub. lic, certified, directory.

Step 3 (performed by a user ${ }^{3}$ wishing to sign a message $m$ ).

- user first calculates the message digest $h-H(m)$.

- user chooses a random $k \in G F *(p)$ such that $(k, p-1)-1$

( $k$ is essentially a session key).

- user calculates $r-\alpha^{k} \bmod p$.

- user calculates $s-k^{-1}(h-x r) \bmod (p-1)$ where $k \cdot k^{-1}=1 \bmod (p-1)$.

Note: The triple $(m ; r, s)$ is the signed message.

A receiver (verifier) on receiving a triple (프, $\underline{\underline{s}}$ ) purporting to be a mes. sage signed by the user whose public key in the directory is y carries out Step 4.

Step 4 (performed by a receiver (verifier)).

- verffier first calculates the message digest $b-H(\mathbb{E})$.

- verifier calculates $u=a^{b}$

- verifier calculates $v-y^{\underline{r}} x^{s}$ mod $p$.

- The triple (mir,s) is accepted as having been signed by the user (associated with the public key $y$ ) if and only if $u-v$.

\section{Appendix B}

The U.S. digital signature algorithm (DSA) $[2,3]$ Involves four distinct steps.

Step 1 (performed by a trusted issuing authority).

- a large (512.1024 bits in 64-bit fncrements) prime, $p$, is randomly selected, subject to the condition that $p$ is divisible by a 160-bit prime, $q$.

- an element, 8 , of order $q$ in $G F(p)$ is constructed by choosing any element $h<G F *(p)$ for which $g-h(p-1) / q>1$.

Note: $p, q$ and $g$ are made public and $c$ an be used by a community of users.

Step 2 (performed by each user who wishes to be able to sign messages).

- user chooses a random $x \in G F^{*}(p)$, which is his secret (signing) key.

- user publishes $y=g^{x}$ mod $p$ as his public (verification) key.

3. The user must bove entered his publit key into the public directory before recelvers can verity his ilenoture. 
Note: each user's public key, $y$, is associated with his identity in a public, certified, directory.

Step 3 (performed by a user" wishing to sign a message m).

- user first calculates the message digest $h=H(m)$.

- user chooses a randora $k \in G F *(q)$.

( $k$ is essentially a session key.)

- user calculates $r=\left(g^{k} \bmod p\right) \bmod q$.

- user calculates $s=k^{-1}(h+x r) \bmod q$. where $k k^{-1}=1 \bmod q$.

Note: The triple $(m ; r, s)$ is the signed message.

A receiver (verifier) on receiving a triple $(\underline{m} ; \underline{\underline{q}}, \underline{s})$ purporting to be a message signed by the user whose public key in the directory is $y$ carries out Step 4.

Step 4 (performed by a receiver (verifler)).

- verifier first calculates the message digest $\mathrm{b}-\mathrm{H}(\mathrm{m})$.

- verifier calculates $t=\underline{s}^{-1} \bmod q$.

- verifier calculates $u_{1}=$ ht mod $q$.

- verifier calculates $u_{2}=$ It mod $q$.

- verifier calculates $v=\left(g^{u_{1}} y^{u_{2}} \bmod p\right) \bmod q$.

- The triple (m; $r, s)$ is accepted as having been signed by the user (associated with the public key $y$ ) if and only if $r-v$.

\section{Reterences}

1. El Gamal, T., "A Public Key Cryptosystem and a Signature Scheme Based on Discrete Logarithms," IEEE Trans, on Info. Theory, Vol. IT-31, No. 4, July 1985, PP. 469-72.

2. NIST, "A Proposed Federal Information Processing Standard for Digital Signal Standard (DSS)." Eed. Register, Vol. 56, No. 169, Aug., 1991, PP. 42980-2.

3. NIST, "Specifications for a Digital signature Standard (DSS), "Federal Information Processing Standards Pub. xx (Draft). Aug. 19, 1991, 12 pps.

4. Simmons, C. J., "The subliminal Channel and Digital signatures." Eurocrypt'84, Paris, France, April 9.11, 1984, Advances in Cryptology, Ed. by $T$. Beth et al., Springer Verlag, Berlin, 1985, pp. 364-378.

5. Simons, G. J., "A Secure Subliminal Channel (?)," Crypto'85, Santa Barbara, $\mathrm{CA}$, August 18-22, 1985, Advances in Croptolegy, Ed. by H. C. Williams, Springer-Verlag, Berlin, 1986, pp. 33-41.

6. Simmons, G. J.. "The Subliminal Channels in the U.S. Digital Signature Algorithm (DSA)." presented at the 3rd Symposium on State and Progress of Research in Cryptography, Rome, Italy, February 15.16, 1993, to be published in the Proceedings of the SPRC' 93.

7. Simmons, G. J., and D. Holdridge, "Forward Search as a Cryptanalytic Tool Against a Public Key Privacy Channel," Proc of the IEEE Computer Soc. 1982 Symp. On Security and Privacy, Oakland, CA, April 26-28, 1982, Pp. 117-128. 